\title{
ORIGINAL ARTICLE Genomic disruption of the histone methyltransferase SETD2 in chronic lymphocytic leukaemia
}

\author{
H Parker ${ }^{1,15}$, MJJ Rose-Zerilli ${ }^{1,15}$, M Larrayoz ${ }^{1,15}$, R Clifford ${ }^{2}$, J Edelmann ${ }^{3}$, S Blakemore ${ }^{1}, J_{\text {Gibson }}^{4}$, J Wang ${ }^{5}$, V Ljungström ${ }^{6}$, TK Wojdacz ${ }^{1}$,

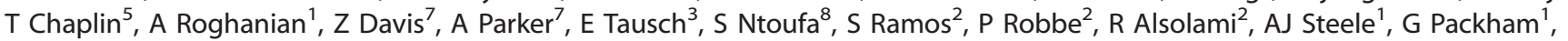

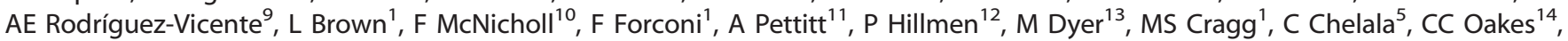 \\ R Rosenquist ${ }^{6}$, K Stamatopoulos ${ }^{8}$, S Stilgenbauer ${ }^{3}$, S Knight ${ }^{2}$, A Schuh $^{2}$, DG Oscier ${ }^{1,7}$ and JC Strefford ${ }^{1}$
}

Histone methyltransferases (HMTs) are important epigenetic regulators of gene transcription and are disrupted at the genomic level in a spectrum of human tumours including haematological malignancies. Using high-resolution single nucleotide polymorphism (SNP) arrays, we identified recurrent deletions of the SETD2 locus in 3\% (8/261) of chronic lymphocytic leukaemia (CLL) patients. Further validation in two independent cohorts showed that SETD2 deletions were associated with loss of TP53, genomic complexity and chromothripsis. With next-generation sequencing we detected mutations of SETD2 in an additional $3.8 \%$ of patients (23/602). In most cases, SETD2 deletions or mutations were often observed as a clonal event and always as a mono-allelic lesion, leading to reduced mRNA expression in SETD2-disrupted cases. Patients with SETD2 abnormalities and wild-type TP53 and ATM from five clinical trials employing chemotherapy or chemo-immunotherapy had reduced progression-free and overall survival compared with cases wild type for all three genes. Consistent with its postulated role as a tumour suppressor, our data highlight SETD2 aberration as a recurrent, early loss-of-function event in CLL pathobiology linked to aggressive disease.

Leukemia (2016) 30, 2179-2186; doi:10.1038/leu.2016.134

\section{INTRODUCTION}

The transfer of methyl groups from S-adenosyl methionine to lysine or arginine residues on histone proteins, catalysed by histone methyltransferases (HMTs), is an important regulator of gene transcription. Accordingly, HMTs are disrupted by various mechanisms, including chromosomal translocations, genomic loss and/or point mutations in both solid and haematological malignancies. ${ }^{1}$ Among the increasing number of HMT aberrations identified in human malignancies, recurrent loss and/or inactivating mutations of the tumour suppressor gene SETD2 were initially identified in clear cell renal cell carcinoma ${ }^{2}$ and subsequently in other solid tumours, for example, high-grade gliomas. ${ }^{3}$ Moreover, SETD2 mutations have been reported in a subset of patients with acute lymphoblastic leukaemia ${ }^{4}$ and acute myeloid leukaemia, especially those with rearrangements in the another HMT gene, $M L L .^{5}$ SETD2 is the only enzyme that catalyses the trimethylation of lysine 36 on histone 3 (H3K36me3), one of the major chromatin marks associated with active transcription. Recent studies have linked SETD2 to the maintenance of genomic integrity through coordination of homologous recombination repair after double strand breaks. The loss of SETD2 impairs DNA repair and enhances genomic instability, supporting its tumour suppressor role. ${ }^{6-9}$

Chronic lymphocytic leukaemia (CLL) is characterized by remarkable clinical heterogeneity such that some patients pursue an indolent course while others require early treatment. Considerable effort has focused on understanding the genetic diversity that underpins this clinical heterogeneity. High-resolution genomic arrays and next-generation sequencing have identified recurring novel regions of genomic copy-number aberrations (CNAs) like del(13q), del(11q), trisomy 12 and del(17p) and recurrent driver mutations in genes such as TP53, ATM, SF3B1 and NOTCH1, respectively (reviewed in Guièze and $\mathrm{Wu}^{10}$ ). Mutations frequently involve genes encoding proteins with important roles in cell signalling, cell cycle control, DNA repair and RNA splicing and processing; however the reported incidence of mutations in chromatin modifiers is lower than in many other haematological malignancies.

In this study, we report the identification of recurrent deletions and mutations of the SETD2 gene in large, well-characterized CLL cohorts. SETD2 lesions appear to represent early events in CLL pathogenesis, often coexisting with, but preceding TP53 abnormalities. They are associated with genomic complexity and

\footnotetext{
${ }^{1}$ Academic Unit of Cancer Sciences, Faculty of Medicine, Cancer Research UK Centre and Experimental Cancer Medicine Centre, University of Southampton, Southampton, UK; ${ }^{2}$ Oxford National Institute for Health Research, Biomedical Research Centre/Molecular Diagnostic Centre, University of Oxford, Oxford, UK; ${ }^{3}$ Department of Internal Medicine III, Ulm University, Ulm, Germany; ${ }^{4}$ Centre for Biological Sciences, Faculty of Natural and Environmental Sciences, University of Southampton, Southampton, UK; ${ }^{5}$ Centre for Molecular Oncology, Barts Cancer Institute, Queen Mary's, University of London, London, UK; ${ }^{6}$ Department of Immunology, Genetics and Pathology, Science for Life Laboratory, Uppsala University, Uppsala, Sweden; ${ }^{7}$ Department of Molecular Pathology, Royal Bournemouth Hospital, Bournemouth, UK; ${ }^{8}$ Institute of Applied Biosciences, Center for Research and Technology Hellas, Thessaloniki, Greece; ${ }^{9}$ Department of Haematology, University Hospital of Salamanca-Biomedical Research Institute of Salamanca, IBMCC, Comprehensive Cancer Center Research, University of Salamanca-CSIC, Salamanca, Spain; ${ }^{10}$ Department of Haematology, Altnagelvin Area Hospital, Western Health and Social Care Trust, Londonderry, UK; ${ }^{11}$ Department of Molecular and Clinical Cancer Medicine, Royal Liverpool and Broadgreen University Hospitals NHS Trust, Liverpool, UK; ${ }^{12}$ Department of Haematology, St James's University Hospital, Leeds, UK; ${ }^{13}$ College of Medicine, Biological Sciences and Psychology, University of Leicester, Leicester, UK and ${ }^{14}$ Division of Hematology, Department of Internal Medicine, The Ohio State University, Columbus, OH, USA. Correspondence: Professor JC Strefford, Cancer Genomics Group, Cancer Sciences Division, Southampton General Hospital, Somers Cancer Research Building, Tremona Road, Southampton SO16 6YD, UK.
}

E-mail: JCS@soton.ac.uk

${ }^{15}$ These authors contributed equally to this work.

Received 2 November 2015; revised 27 April 2016; accepted 3 May 2016; accepted article preview online 20 May 2016 ; advance online publication, 10 June 2016 
chromothripsis, and identify a subgroup of patients with poor outcome.

\section{PATIENTS AND METHODS}

\section{Patients}

We studied samples taken from 1006 CLL patients either at entry into one of five clinical trials or from a cohort of untreated patients with progressive disease managed at the Royal Bournemouth Hospital. Four randomized trials (ADMIRE, ARCTIC, UK CLL4 ${ }^{\text {(ref. } 11)}$ and GCSG CLL8 ${ }^{\text {(ref. } 12)}$ ) compared chemo or chemo-immunotherapy regimens in fit previously untreated patients while the fifth trial (SCSG CLL2O) enroled ultra-high-risk patients who were either refractory to a purine analogue or were previously untreated with a $17 p$ deletion. Further details of the clinical trials are provided in Supplementary Table S1. All patients were diagnosed using standard morphologic and immunophenotypic criteria. Informed consent was obtained from all patients in accordance with the Helsinki declaration, and this study was approved by national or regional research ethics committees.

Patients were grouped into three cohorts (discovery $(n=261)$, extension $(n=635)$ and ultra-high-risk $(n=110))$; details of the cohort composition and SETD2 analysis are summarized in Table 1, Supplementary Methods and Supplementary Figure S1. DNA was extracted from CLL B-cell samples (all with $>80 \%$ tumour purity) and from matched germline DNA for SETD2-mutated cases as outlined in Supplementary Methods. The assessment of established biomarkers was performed as previously described. ${ }^{13}$ In total, 572 and 602 samples were screened for SETD2 loss and mutation, respectively, with 168 cases screened for both loss and mutation.

Genome-wide microarray-based copy-number analysis

DNA from 261 discovery and 110 ultra-high-risk cases was amplified, labelled and hybridized to the Affymetrix SNP6.0 platform, aligned onto the human genome sequence (GRCh37) and analysed in Partek Genomics Suite (Partek, St Louis, Inc., MO, USA) as reported previously. ${ }^{14-18}$ DNA from 201 pretreatment extension cases (ADMIRE and ARCTIC) was hybridized to the Illumina HumanOmni1-Quad and HumanOmniS-8 platforms according to the manufacturer's protocols. ${ }^{19,20}$ Further experimental details are provided in the Supplementary Methods.

Targeted re-sequencing and whole-exome sequencing

Ninety-three CLL samples from the discovery cohort (and five matched germline controls) were processed and analysed for mutations in SETD2 (all exons) and a number of clinically relevant genes with a bespoke Haloplex Target Enrichment system (Agilent Technologies, Santa Clara, CA, USA) (Supplementary Methods and Table 1) and processed and analysed as previously reported. ${ }^{21}$ An additional 231 cases from our pre-treatment extension cohort were screened for SETD2 mutations using a TruSeq Custom Amplicon panel (lllumina Inc., San Diego, CA, USA) as previously described. ${ }^{20,22}$ All the variants identified by both platforms were annotated against dbSNP (build 135) and functional prediction was also performed using SIFT andPolyphen2 analysis. Somatically acquired SETD2 mutations $(n=4)$ were also identified in the recent whole-exome sequencing study of 278 matched tumour and germline cases from the GCSG CLL8 study. ${ }^{23}$ Additional experimental details are provided in the Supplementary Methods.

\section{Sanger validation}

Variants in SETD2 were subjected to validation by conventional Sanger-based sequencing of PCR products obtained from tumour $(n=11)$ and where possible, paired normal genomic DNA $(n=5)$. The expression of SETD2 mutations at mRNA level was also tested in samples with available material $(n=4)$. Primers for DNA or mRNA validation are listed in Supplementary Table S2.

\section{Quantitative reverse transcriptase-PCR}

Total RNA was isolated from purified CLL cells of 36 patient samples using RNeasy columns (Qiagen, Manchester, UK) and reversed transcribed using the Improm II RT-PCR kit (Promega, Southampton, UK) according to the manufacturer's instruction. Primers and probes for the housekeeping genes (18 s) and target genes (CCDC12, NBEAL2, KIF9, KLHL18, SETD2) were selected using the Universal Probe Library (Roche Applied Science, Burgess Hill, UK) (Supplementary Table S3). Two independent assays were designed to ascertain
Table 1. Cohort characteristics

\begin{tabular}{|c|c|c|c|}
\hline Characteristics & $\begin{array}{l}\text { Discovery, } \\
\text { N (\%) }\end{array}$ & $\begin{array}{l}\text { Extension, } \\
\mathrm{N}(\%)\end{array}$ & $\begin{array}{l}\text { Ultra-high- } \\
\text { risk, N (\%) }\end{array}$ \\
\hline $\begin{array}{l}\text { Number of cases (number } \\
\text { with germline material) }\end{array}$ & $261(5)$ & $635(280)$ & 110 \\
\hline Origin & $\begin{array}{l}\text { CLL4/Local } \\
\text { cohort }\end{array}$ & $\begin{array}{l}\text { CLL4/ARCTIC/ } \\
\text { ADMIRE/CLL8 }\end{array}$ & CLL2O \\
\hline \multicolumn{4}{|l|}{ Treatment naive } \\
\hline Yes & $261(100)$ & $635(100)$ & $35(32)$ \\
\hline No & - & - & $75(68)$ \\
\hline \multicolumn{4}{|l|}{ Gender } \\
\hline Male & $192(74)$ & $336(53)$ & $79(72)$ \\
\hline Female & $69(26)$ & 299 (47) & $31(28)$ \\
\hline \multicolumn{4}{|c|}{ IGHV gene mutational status } \\
\hline Unmutated & $120(46)$ & $350(55)$ & $96(87)$ \\
\hline Mutated & $68(26)$ & $232(37)$ & $12(11)$ \\
\hline No data & $73(28)$ & $53(8)$ & $2(2)$ \\
\hline \multicolumn{4}{|l|}{$\operatorname{del}(17 p)$} \\
\hline Yes & $29(11)$ & $32(5)$ & $88(80)$ \\
\hline No & $214(82)$ & $565(89)$ & $22(20)$ \\
\hline No data & $18(7)$ & $38(6)$ & - \\
\hline \multicolumn{4}{|l|}{$\operatorname{del}(11 q)$} \\
\hline Yes & $79(30)$ & $138(22)$ & $21(19)$ \\
\hline No & $165(63)$ & $461(73)$ & $89(81)$ \\
\hline No data & $17(7)$ & $36(5)$ & - \\
\hline \multicolumn{4}{|l|}{ Tri12 } \\
\hline Yes & $23(9)$ & $58(9)$ & $19(17)$ \\
\hline No & $199(76)$ & $418(66)$ & $91(83)$ \\
\hline No data & 39 (15) & $159(25)$ & - \\
\hline \multicolumn{4}{|l|}{$\operatorname{Del}(13 q)$} \\
\hline Yes & $103(40)$ & $252(40)$ & $65(59)$ \\
\hline No & $63(24)$ & $224(35)$ & $45(41)$ \\
\hline No data & $95(37)$ & $159(25)$ & - \\
\hline Chromothripsis & $8(3)$ & $9(1.4)$ & $10(9)$ \\
\hline SETD2 deleted & 8/261 (3) & 9/201 (4.5) & 10/110 (9) \\
\hline SETD2 mutated & 4/93 (4.3) & $11 / 509(2.2)$ & - \\
\hline $\begin{array}{l}\text { SETD2 deleted with } \\
\text { TP53 abnormalities }\end{array}$ & $3 / 8(37.5)$ & $5 / 9(55.5)$ & $\begin{array}{l}10 / 10 \\
(100)\end{array}$ \\
\hline $\begin{array}{l}\text { SETD2 mutated with } \\
\text { TP53 abnormalities }\end{array}$ & $0 / 4(0)$ & 4/11 (36.4) & - \\
\hline
\end{tabular}

expression of $3^{\prime}$ and $5^{\prime}$ SETD2. Normal B-cell mRNA was use to normalize the expression of each gene by delta-delta CT method as previously described. ${ }^{24}$

Statistical analysis

Statistical analysis was performed with SPSS v22. Differences between samples were analysed by Mann-Whitney U-test. Progression-free survival (PFS) and overall survival (OS) were calculated for clinical trial samples from randomization. Survival analysis was performed by Kaplan-Meier and logrank analysis. Significant differences were considered with $P$-values lower than 0.05 .

\section{RESULTS}

Recurrent deletions of $3 p$ are a feature of CLL

We identified 1024 acquired CNAs (mean 3.9, range 0-45) in our discovery cohort (Supplementary Table S4). Deletions of chromosome $3 p[\operatorname{del}(3 p)]$ were observed in eight patients $(3 \%)$, ranged from 0.45 to $81 \mathrm{Mb}$ in size (Supplementary Table S5) and identified a well delineated minimally deleted region (MDR) between genomic location 46.96 and $47.39 \mathrm{Mb}$, containing the genes CCDC12, NBEAL2, SETD2, KIF9 and KLHL18 (Figure 1a). We 
a

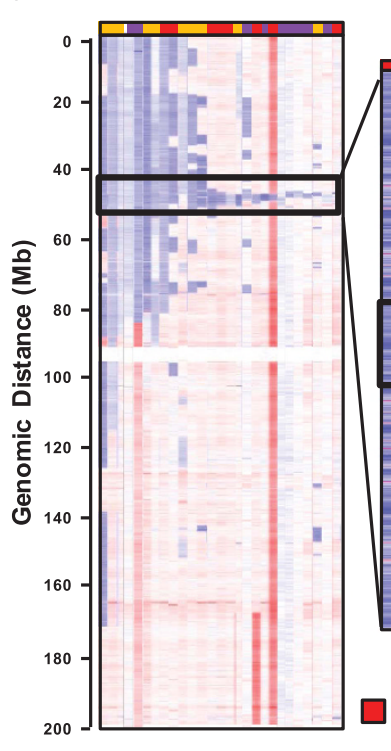

Discovery

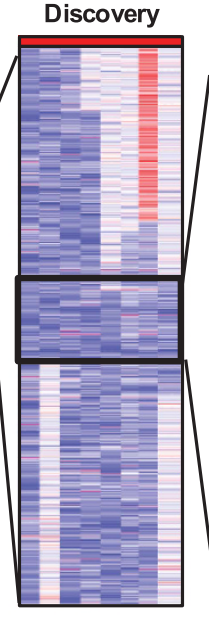

Discovery $8 / 261(3 \%)$

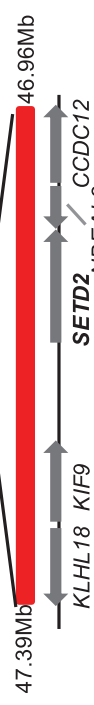

Extension 9/200 (4.5\%)
Extension

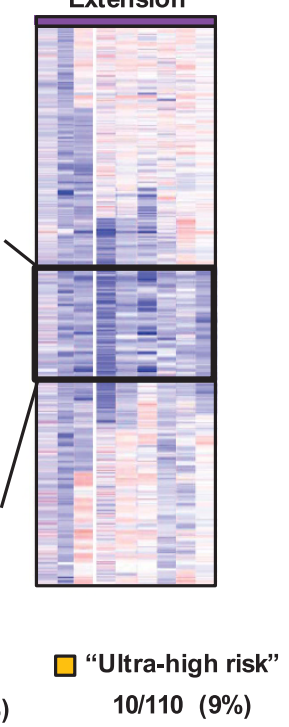

b

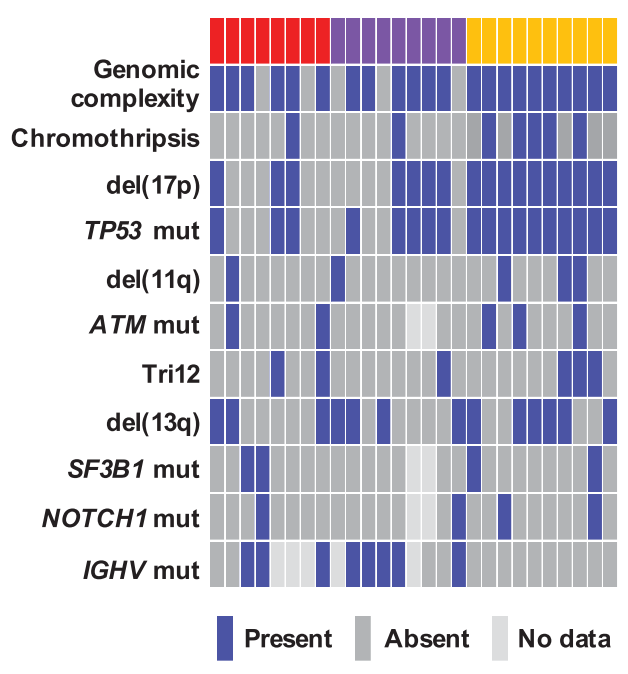

C

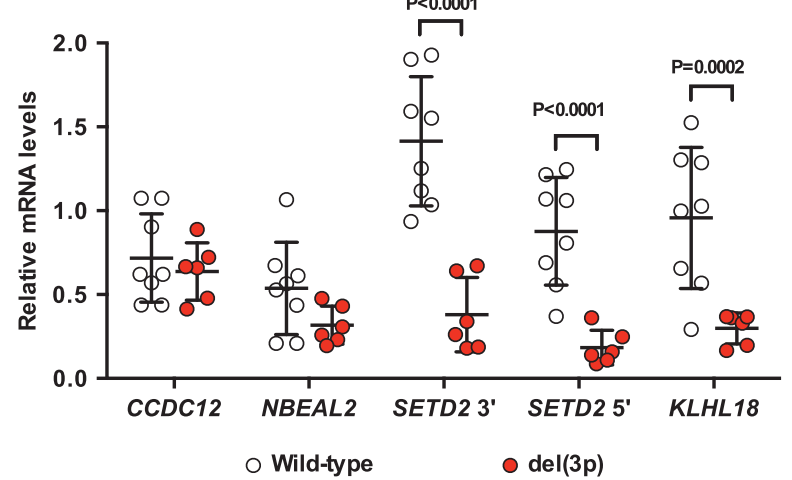

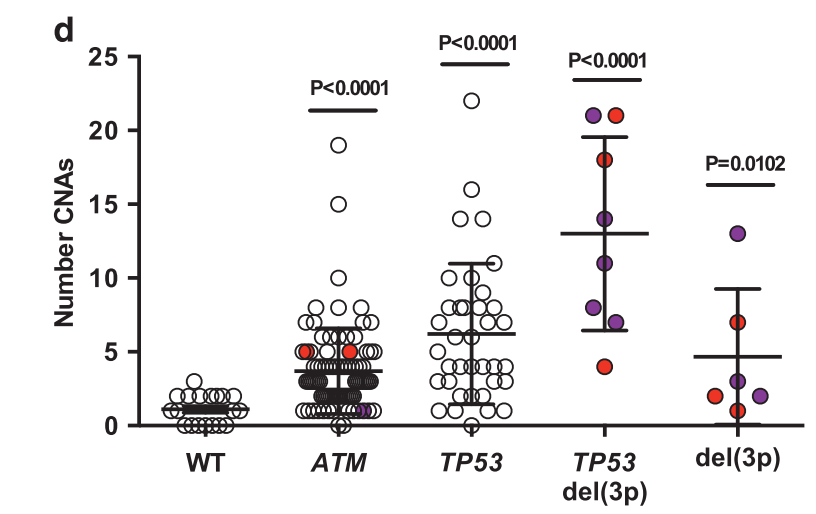

Figure 1. SETD2 deletions in our discovery, extension and ultra-high-risk cohorts. (a) SNP6.0 data for the del(3p) cases. Genomic location is indicated by the ladder to the left. Each column represents one patient. Loss, gain and normal copy number are shown as blue, red and white, respectively. The black box indicates the MDR, and is displayed in greater detail for our discovery and extension cohorts. The genes in the MDR with their transcriptional direction are displayed in the middle, with the MDR from the discovery and extension cohorts shown by the red and purple bars, respectively. (b) Matrix displaying the biomarkers and genomic features associated with del(3p) cases with the discovery, extension and ultra-high-risk cases shown in red, purple and yellow, respectively. (c) Real-time PCR expression for the five genes localized in the discovery MDR in cases with or without del(3p). All the samples were negative for KIF9. $18 \mathrm{~s}$ was employed as housekeeping gene. Expression in normal B-cells was used as a normalization sample. Mean \pm s.d. is represented. (d) Scatterplots displaying the number of CNA observed in subgroups of our cohort (excluding ultra-high-risk cases). Cases were assigned to a subgroup using a hierarchical model; presence of del(17p) and/or TP53 mutation, then del(11q) and/or ATM mutation, then del(3p) cases with and without TP53 abnormalities and then wild-type (WT) cases containing no del(17p), del(11q), del(3p) or mutations in ATM and TP53. Mean \pm s.d. is represented.

compared the expression of these genes by quantitative reverse transcriptase-PCR in $3 p$ deleted $(n=6)$ versus non-3p deleted patients $(n=8)$ (Figure 1c). We were not able to detect the expression of KIF9 mRNA in CLL or normal B-cells. Within the MDR, the HMT gene SETD2 was significantly underexpressed, measuring by two different assays targeting the $3^{\prime}$ or $5^{\prime}$ region of the mRNA $(P<0.0001$ for both assays).

We then aimed to confirm the presence of $3 p$ deletions and refine the MDR in our extension cohorts. Firstly, we identified nine del(3p) cases (4.5\%) in our extension pre-treatment cohort, permitting the MDR to be refined to the SETD2 and KIF9 loci (47.12-47.36 Mb; Figure 1a and Supplementary Table S5). Across our discovery and pre-treatment extension cohorts, SETD2 deletions were present in $17 / 461$ cases (3.7\%), significantly associated with deletions and/or mutations of TP53 $(P=0.003)$ and genomic complexity ( $\geqslant 3$ deletions, ${ }^{25} P=0.04$ ) (Figure $1 \mathrm{~b}$ ). GISTIC 2.0 analysis, $^{26}$ an algorithm for identifying statistically significant regions of CNA above an estimated background rate (FDR $q$-value $<0.25$ ), showed that in 39 TP53 deleted cases (del(3p), $n=15$ ), the SETD2 region on $3 p 21.31$ was deleted at a significant frequency $(q$-value $=0.001)$, ranked third after del $(13 q)$ and del (17p) (Supplementary Figure S2).

Interestingly, SETD2 deletions without concomitant TP53/ATM abnormalities $(n=6)$ also exhibited significantly more genomic complexity than wild-type patients $(P=0.01$; Figure $1 \mathrm{~d})$. Two SETD2-deleted cases showed evidence of chromosome 3 chromothripsis (based on $>10$ CNAs per chromosome ${ }^{18}$ ) (Figures 1a and b). In the ultra-high-risk cohort, SETD2 deletions were detected in $9 \%$ of cases (10/110), and were significantly enriched compared with the pre-treatment cohort $(P=0.009)$. All 10 had loss of TP53 and 5 had concomitant chromosome 3 chromothripsis (Figure 1b). To further establish the significance of our SETD2 deletion in cases with chromosome 3 chromothripsis, we mapped all recurrently $3 p$ deletions in these cases. This analysis 
showed that while additional regions of recurrent deletion were observed on $3 p$, the only regions shared across all patients included the SETD2 locus (Supplementary Table S6).

We analysed SETD2 expression in an extended cohort of patients with $3 p$ deletions $(n=16)$, and again the expression was diminished in these patients compared with wild-type patients $(P=0.0068$; Supplementary Figure S3). In order to study the clonal nature of the SETD2 deletions, we assigned each genomic CNA with a relative copy-number value by normalizing CNA intensity values from array features. We excluded regions with gain and sex chromosome CNAs from the analysis. The cutoff for normal copy number was established between 1.7 and 2.3. We could infer that the $3 p$ deletion was in the dominant clonal population in 11/18 (61\%) cases with data available for analysis (Supplementary Figure S4).

\section{SETD2 mutations in CLL}

To identify somatic gene mutations, we initially employed targeted re-sequencing of 93 discovery cohort cases and identified 122 nonsilent mutations (non-synonymous $n=80$, frameshift indel $n=20$, splicing $n=9$, nonframeshift indel $n=6$, stopgain $n=6$, stoploss $n=1$ ) targeting 37 genes in $71 / 93$ cases (mean 1.8, range 1-4). Sanger sequencing confirmed $93.6 \%$ of the tested variants $(n=79)$, while the remaining unconfirmed variants were present at low read depth $(n=3)$ or in a low percentage of mutant reads $(n=2)$. We found ATM $(n=14)$, TP53 $(n=14)$, NOTCH1 $(n=20)$ and SF3B1 $(n=15)$ mutations at a frequency expected for the studied cohort, which aligns with published data and demonstrates the validity of the re-sequencing platform. We identified non-synonymous SETD2 mutations in four (4.3\%) discovery cases (p.D99G, p.Q1545K, p.W1306*, p.E1955Q) (Figures 2a and b). Sanger sequencing validated that all of the SETD2 mutations were present in tumour DNA. We obtained matched germline DNA from three patients and confirmed that the mutations were somatically acquired (p.D99G, p.W1306*, p.E1955Q) (Supplementary Figure S5A).

To corroborate this preliminary observation, we investigated 231 cases of our pre-treatment extension cohort by TruSeq ampliconbased sequencing. We identified an additional nine (3.9\%) SETD2 mutations (p.A50T, p.L89F, p.P167L, p.N535S, p.E670K, p.M1742L, p.M1889T (x2), p.I2295M) (Figures 2a and b). Sanger sequencing confirmed each SETD2 variant in the tumour material and in two cases with germline material available, the variants were somatically acquired. Assessment of whole-exome sequencing data of the CLL8 study ${ }^{23}$ samples included in our pre-treatment extension cohort revealed the presence of somatically acquired SETD2 mutations in 4/278 cases (1.4\%), namely, p.EEEELQSQQ1919fs, p.L1804fs, p.VLEYC1576del, p.V1190M. None of these SETD2 mutations (Table 2) are annotated in COSMIC. ${ }^{27}$ During the preparation of this manuscript, a study performed by Puente et al. ${ }^{28}$ in $506 \mathrm{CLL}$ patients also described both SETD2 mutations (0.8\% of cases) and deletions in $3 p$ ( $2 \%$ of cases) whose MDR encompassed SETD2 while Landau et al. ${ }^{23}$ identified SETD2 mutations in 8/538 (1.5\%) cases.

In total, across our cohorts there were 15 somatically acquired SETD2 variants $(15 / 602 ; 2.5 \%)$. An additional eight variants that could not be examined in germline material were either absent $(n=3)$, reported to have a very low prevalence $(n=5)$ in 1000 Genomes project or have a subclonal variant allele frequency (\%VAF $<0.45(n=1)$; Table 2). Therefore, while these eight variants are predicted to be functionally deleterious, we cannot exclude that the minority may be rare germline variants as they exhibit clonal variant allele frequencies in the tumour material.

We were able to confirm the expression of the SETD2 mutations at mRNA level in four of our patients with available material (p.D99G, p.Q1545K, p.E1955Q, p.E670K) (Supplementary Figure $S 5 A$ ), and quantiative reverse transcriptase-PCR analysis of three SETD2 mutated samples showed that SETD2 mRNA expression was reduced compared with wild-type patients $(P=0.035$; Supplementary Figure S3).
We performed integrative analysis of 93 cases from our discovery cohort with Haloplex re-sequencing and SNP6.0 copy-number data available, by employing the ABSOLUTE algorithm. ${ }^{29}$ This approach estimates the cancer cell fraction harbouring a given mutation by correcting for sample purity and local copy-number changes. Mutations were classified as clonal if the cancer cell fraction was $>0.95$ with a probability $>0.5$ and subclonal otherwise. ${ }^{30}$ In additional cases with proven-somatic SETD2 mutations $(n=4)$ and paired copy-number data from our pre-treatment validation cohorts, we performed this estimation by manually correcting for tumour sample purity and local copy number. Our analysis demonstrated the expected subclonal distribution of established gene mutations, such as TP53, ATM, SF3B1 and NOTCH1. Interestingly, all our somatically acquired SETD2 mutations exhibited a clonal cancer cell fraction, suggesting that these mutations may be early events in the evolution of CLL (Figures $2 \mathrm{c}$ and $\mathrm{d}$ and Supplementary Figures S5B and C), although further studies are required to confirm this observation.

\section{SETD2 aberrations are associated with inferior progression-free} and OS

Finally, we analysed the impact of SETD2 abnormalities (deletion or somatically acquired mutation) on PFS and OS in front-line trial patients. We observed a significantly shorter PFS in cases with SETD2 abnormalities that were wild type for TP53/ATM $(n=7)$, compared with cases wild type for TP53/ATM/SETD2 $(n=62)$ (PFS: 30 vs 48 months; $P=0.003$ ) (Figure $2 \mathrm{e}$ ). The same patients with SETD2 abnormalities $(n=7)$ also had a shorter OS than wild-type patients ( $n=62$ ) (OS: 34 vs 92 months; $P<0.001$ ) (Figure 2f). While these data suggest that SETD2 aberration may be clinically relevant, further investigation in larger cohorts is needed to understand their full impact on survival.

\section{DISCUSSION}

This study was based on an initial high-resolution SNP6.0 array analysis of 261 untreated patients with progressive CLL, which identified a recurrent deletion of the short arm of chromosome 3 in $3 \%$ of cases $(n=8)$. The MDR included the CCDC12, NBEAL2, SETD2, KIF9 and KLHL18 genes, of which SETD2 was the most significantly underexpressed in tumour cells. We then identified clonal, somatically acquired SETD2 mutations in $4.3 \%$ of this cohort; no mutated case had a concomitant SETD2 deletion.

The SETD2 gene encodes a $230 \mathrm{kDa}$ protein that is non-redundantly responsible for all trimethylation of lysine 36 on histone H3 (H3K36me3), ${ }^{31,32}$ a mark that is associated with actively transcribed regions and is involved in transcriptional elongation and splicing. $^{33}$ In addition, recent studies have linked this epigenetic histone mark to other important cellular processes such as the regulation of mismatch repair, efficient homologous recombination and the maintenance of genomic stability. ${ }^{7-9}$ In vitro inhibition of Setd2 decreases global levels of H3K36me3 and impairs the recruitment of the mismatch recognition protein hMutSa onto chromatin, thereby preventing appropriate DNA mismatch repair. Cells lacking the Setd 2 protein display microsatellite instability and have elevated levels of spontaneous mutations. ${ }^{7,34-36}$ Inactivating SETD2 mutations were first described in clear cell renal cell carcinoma, ${ }^{2,6}$ subsequently in other solid tumours such as paediatric high-grade gliomas and most recently in a subset of patients with acute lymphoid and myeloid leukaemias. ${ }^{2,5,37,38}$ SETD2 mutations in clear cell renal cell carcinoma are frequently associated with $3 p$ deletions resulting in loss of both SETD2 and VHL genes, while in acute leukaemias, SETD2 mutations may be bi-allelic but $3 p$ loss is rare. SETD2 genomic abnormalities are associated with decreased H3K36me3 levels, a distinctive DNA methylation signature $^{6}$ and chemoresistance in paediatric acute lymphoblastic leukaemia. ${ }^{39}$ In MLL-rearranged cells from acute leukaemic patients, 


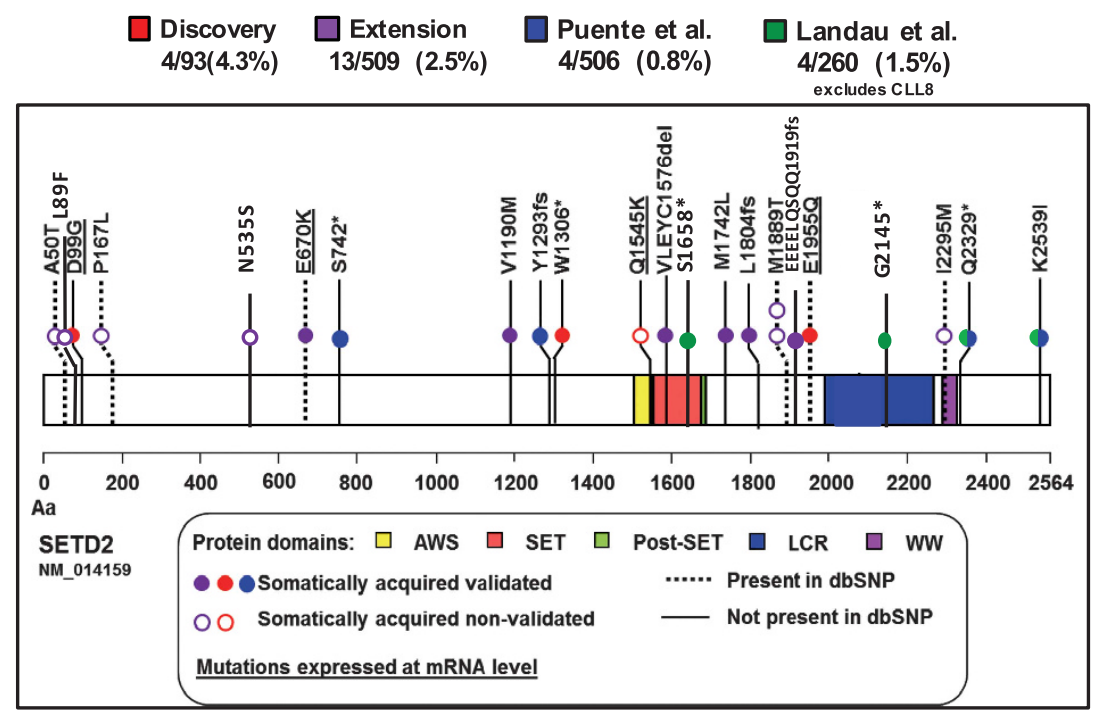

Discovery 4/93(4.3\%)
Extension $3 / 509$ (2.5\%)
Puente et al. $4 / 506(0.8 \%)$ b

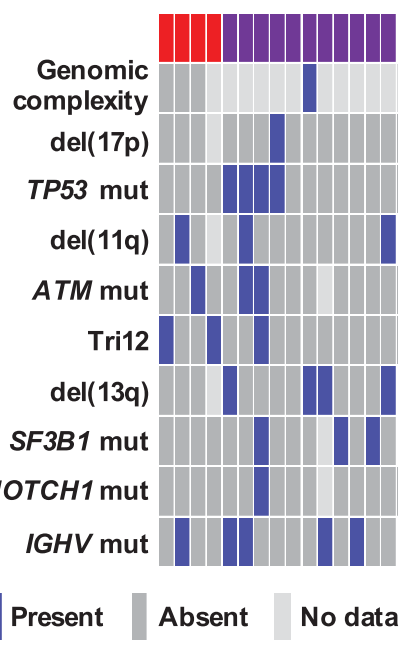

C

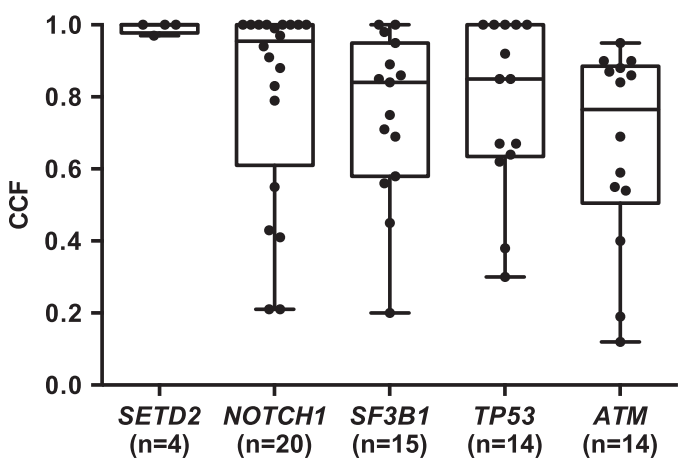

e

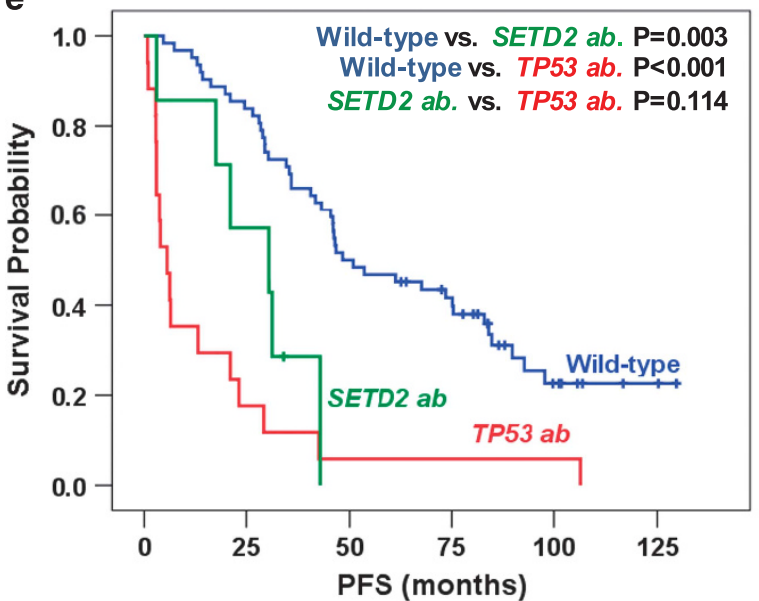

d

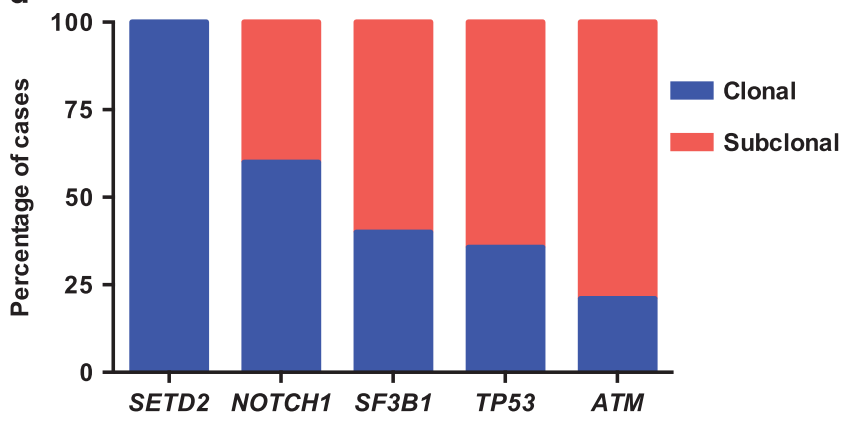

f

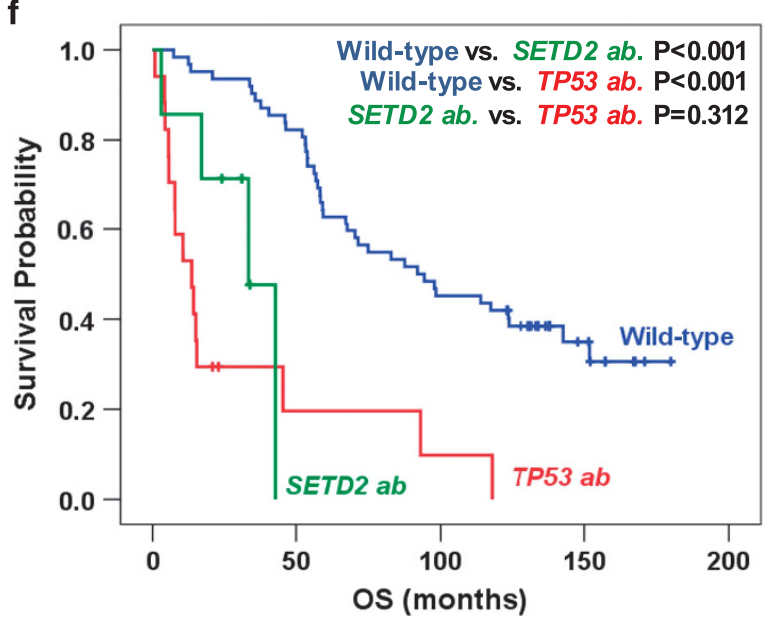

Figure 2. SETD2 mutations in our discovery and extension cohorts. (a) Schematic diagram of the Setd2 protein with their key functional domains. Mutations are displayed in the diagram. The colour denotes the cohort, and the filled circles are mutations that have been confirmed as somatically acquired. (b) Matrix displaying the biomarkers and genomic features associated with SETD2-mutated cases in the discovery (red) and extension (purple) cases. (c) Analysis of the clonality for SETD2 and other recurrently mutated genes on CLL. For each case the cancer cell fraction (CCF) is derived manually or with the ABSOLUTE algorithm. Only somatically acquired validated mutations are displayed (cases 69, 100, S21 and 88). The number of mutations ( $n$ ) for each gene in the analysis is shown (bottom). (d) Percentage of cases harbouring clonal or subclonal mutations for each of the genes displayed. (e) Kaplan-Meier and log-rank analysis for progression-free survival (PFS) in patients carrying SETD2 abnormalities ('SETD2 ab') but wild type for TP53 or ATM deletion and/or mutation compared with those with TP53 abnormalities ('TP53 ab') and those wild type for TP53, ATM and SETD2 ('wild type'). (f) Kaplan-Meier and log-rank analysis for overall survival (OS) in the same categories described in (e). 


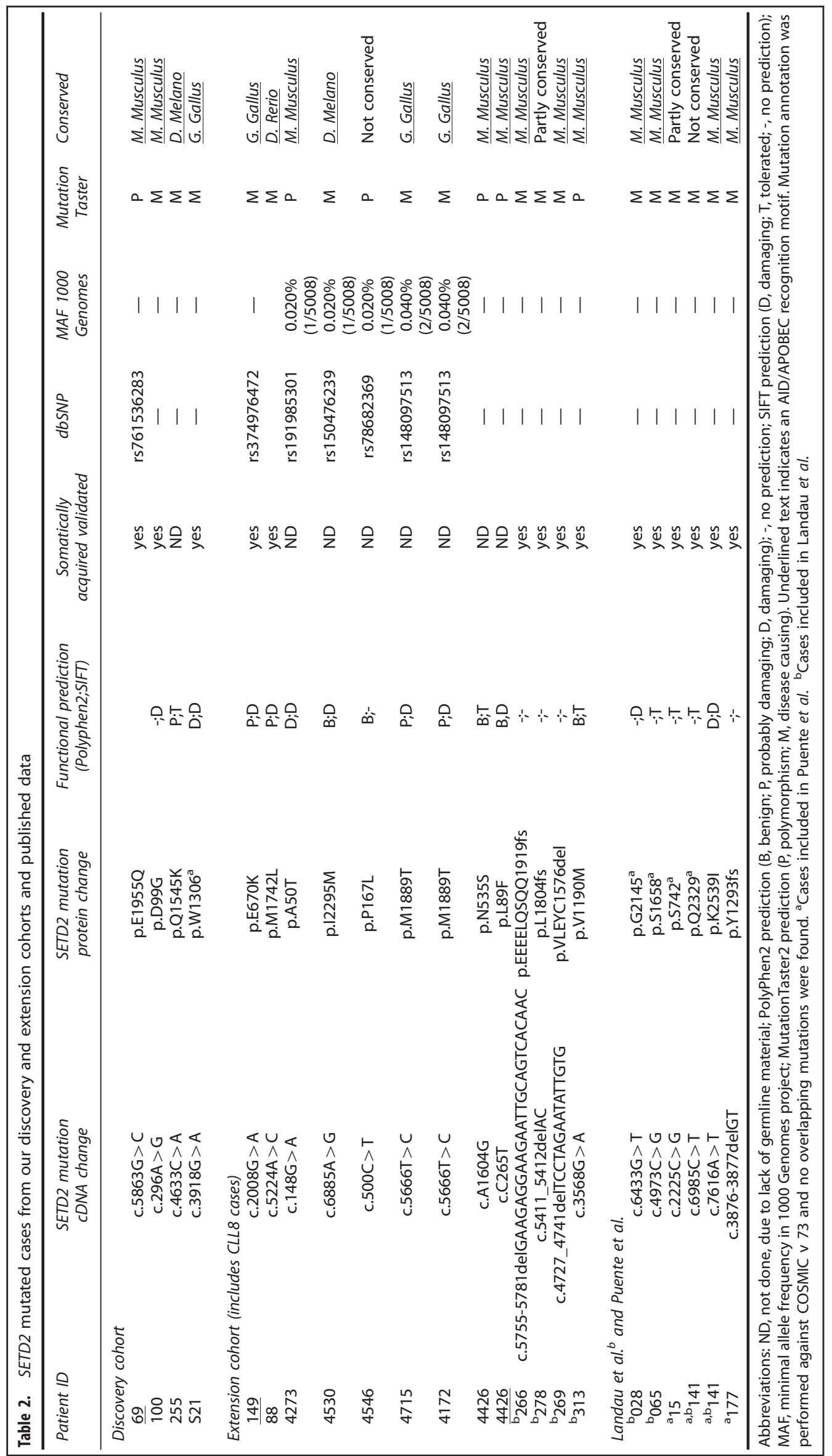


Setd 2 knockdown is implicated in disease initiation and progression by promoting the self-renewal capacity of leukaemic stem cells.

In view of the role of SETD2 disruption in tumorigenesis and the identification of SETD2 abnormalities in our discovery cohort, we then accrued samples from other patient cohorts, including the GCLLSG CLL8 cohort in which $3 p$ deletions had also been detected, ${ }^{18}$ to confirm the incidence of SETD2 disruption and evaluate its biological and clinical consequences in CLL. Previously untreated patients sampled at randomization to chemo or chemoimmunotherapy trials had a similar incidence of $3 p$ deletions $(4.5 \%)$ to that seen in the discovery cohort while a higher incidence of loss (9\%) was found in the ultra-high-risk cohort. The inclusion of additional cohorts enabled a smaller MDR to be defined, including SETD2 and KIF9, implicating SETD2 as the key deleted gene. The incidence of SETD2 mutations was comparable in all cohorts tested, no synonymous mutations were identified and when germline material was tested, all mutations were somatically acquired. The diverse sequencing strategies utilized in this current study precluded the application of computational tools like MutSigCV, ${ }^{40}$ an algorithm that identifies significantly mutated genes by accounting for background mutation rate, DNA replication time and the gene size. However, we did assess the SETD2 background mutation rate, expression level and replication timing data from Lawrence et al. ${ }^{40}$ demonstrating that SETD2 shares no properties associated with false-positive candidate cancer genes (Supplementary Figure S7). The recent studies by Puente et al. and Landau et al. published during the preparation of this manuscript confirm the rare but recurrent nature of SETD2 abnormalities. ${ }^{23,28}$ SETD2 deletions were not over-represented by analysis of whole-exome sequencing generated copy-number data in the work by Landau and the mutation frequencies of both studies were lower than those in our study. The different frequencies reported in these two studies could be explained by cohort composition, as our study included ultra-high-risk CLL and patients randomized to clinical trials.

As we found SETD2 mRNA expression to be downregulated in cases with either SETD2 deletion or mutations and as we did not observe bi-allelic SETD2 abnormalities, we assessed whether SETD2 may also be deregulated by DNA methylation. Kulis and co-workers ${ }^{41}$ reported no differential methylation levels in the SETD2 gene body and promoter regions (15 and 9 CpG probes), respectively, between unmutated or mutated CLL or major cytogenetic subtypes and SETD2 mRNA expression was not correlated with gene methylation status (doi:10.1038/ng.2443: Supplementary Tables S5 and S11). ${ }^{41}$ Preliminary analysis of our own unpublished Illumina $450 \mathrm{~K}$ methylation array data also demonstrated no differences between mutated and unmutated CLL for these probes (Supplementary Figure S6). In addition, when we analysed SETD2 expression in a published CLL data set (http://www.ncbi.nlm.nih.gov/geo/query/acc.cgi?acc $=$ GSE2466) ${ }^{44}$ using the Oncomine portal (https://www.oncomine.org), ${ }^{42}$ we observed a heterogeneous pattern. Reduced levels are evident in the minority of patients, which given our observed association between SETD2 deletion and expression could imply gene deletion in those Oncomine samples with low mRNA expression. Together, this suggests that DNA methylation does not play a substantial role in regulating SETD2 expression in B-CLL cells, as previously noted in acute leukaemia. ${ }^{5}$

Across all cohorts, SETD2 deletion was found in both IGHV mutated and unmutated cases but was strongly associated with TP53 loss and mutation, likely accounting for its higher incidence in the ultra-highrisk cohort. We also noted an association with genomic complexity even in cases lacking a TP53 or ATM abnormality, consistent with the role of SETD2 in maintaining genomic stability. Moreover, we identify several SETD2 deletions that appeared to be the result of chromothripsis. The somatically acquired SETD2 mutations showed a comparable genomic distribution to those previously described in other tumours and were predicted to have deleterious functional consequences. Furthermore, their association with significantly reduced mRNA expression in those cases analysed, suggest that they either directly affect mRNA expression or coexist with other defects in transcriptional control at this locus. Interestingly, we did not observe a statistically significant association between SETD2 mutations and TP53 abnormalities or genomic complexity, the implication of which may be differing functional consequences of mono-allelic loss and mutation.

In our study, both SETD2 deletions and mutations often appeared to be clonal and may precede TP53 abnormalities in at least some cases. Setd 2 has been shown to directly regulate the transcription of a subset of genes via cooperation with the transcription factor $\mathrm{p} 53{ }^{43}$ and the link between SETD2 and TP53 is an interesting association worthy of functional validation. It is possible that the SETD2 alterations present in our CLL cases may contribute to further inactivation of p53-mediated checkpoint control, a situation that has been proposed in clear cell renal cell carcinoma. ${ }^{8}$ The low frequency of SETD2 disruption and the association with TP53 abnormalities hinder an accurate assessment of its clinical consequences. Nevertheless, we observed a shorter PFS and OS in patients with SETD2 but no TP53 or ATM abnormalities compared with cases wild type for all three genes. In support of this preliminary clinical observation, it has been shown that $3 p$ deletions in head and neck squamous carcinoma are associated with reduced survival. ${ }^{44}$ Furthermore, the authors showed that the coexistence of a TP53 abnormality with del(3p) decreased survival further, an observation that we could not confirm in our cohort.

In summary, our current study provides the first comprehensive analysis of CNAs and mutations targeting the SETD2 gene in a large cohort of patients with CLL. We find somatic deletions and mutations in $\sim 7 \%$ of CLL patients requiring treatment. These associate with TP53 dysfunction, genomic complexity and chromothripsis and may be early clonal events. Functional studies are now warranted to elucidate the exact biological importance of SETD2 in CLL pathogenesis, but our data add to a growing body of evidence suggesting a role for $\mathrm{H} 3 \mathrm{~K} 36 \mathrm{me} 3$ in tumorigenesis that may be exploited for the development of novel therapeutic approaches.

\section{CONFLICT OF INTEREST}

The authors declare no conflict of interest.

\section{ACKNOWLEDGEMENTS}

We gratefully acknowledge all patients who contributed to this study. This work was funded by Bloodwise $(11052,12036)$, the Kay Kendall Leukaemia Fund (873), Cancer Research UK (C34999/A18087, ECMC C24563/A15581), Wessex Medical Research and the Bournemouth Leukaemia Fund. SS is supported by the Else Kröner-FreseniusStiftung (2012_A146) and Deutsche Forschungsgemeinschaft (SFB 1074 projects B1, B2). The LRF CLL4 trial was funded by a core grant from Leukaemia and Lymphoma Research. DG and DC acknowledge the support by The Royal Marsden Hospital and The Institute of Cancer Research National Institute of Health Research Biomedical Research Center. RR is supported by the Swedish Cancer Society, the Swedish Research Council, Science for Life Laboratory, Uppsala University, Uppsala University Hospital, and the Lion's Cancer Research Foundation, Uppsala.

\section{AUTHOR CONTRIBUTIONS}

HP, MJJR-Z, ML, RC, JE, SB, TC, AR, AP, TW, SN, MC, RA, SR, CCO, SK, AR, BY and $\mathrm{LB}$ performed the experimental work; AP performed the molecular diagnostic assays; MJJR-Z, JG, JW, PR, VL and SK conducted the statistical and bioinformatic analyses; FN, FF, AP, PH, MD, SN, TW, CCO, KS, SS, RR, AS and DO contributed patient samples and data; JCS initiated and designed the study; HP, MJJR-Z, DGO, ML and JCS wrote the paper with contributions from RC, ML, GP, AJS, TW, $\mathrm{CCO}, \mathrm{RR}, \mathrm{KS}, \mathrm{AS}$; and all authors critically reviewed the final paper. 


\section{REFERENCES}

1 Baylin SB, Jones PA. A decade of exploring the cancer epigenome-biological and translational implications. Nat Rev Cancer 2011; 11: 726-734.

2 Dalgliesh GL, Furge K, Greenman C, Chen L, Bignell G, Butler A et al. Systematic sequencing of renal carcinoma reveals inactivation of histone modifying genes. Nature 2010; 21: 360-363.

3 Fontebasso AM, Schwartzentruber J, Khuong-Quang DA, Liu XY, Sturm D, Korshunov A et al. Mutations in SETD2 and genes affecting histone H3K36 methylation target hemispheric high-grade gliomas. Acta Neuropathol 2013; 125: 659-669.

4 Zhang J, Ding L, Holmfeldt L, Wu G, Heatley SL, Payne-Turner D et al. The genetic basis of early T-cell precursor acute lymphoblastic leukaemia. Nature 2012; 481: 157-163.

5 Zhu $X$, He F, Zeng $H$, Ling $S$, Chen A, Wang $Y$ et al. Identification of functional cooperative mutations of SETD2 in human acute leukemia. Nat Genet 2014; 46: 287-293.

6 Duns G, van den Berg E, van Duivenbode I, Osinga J, Hollema H, Hofstra RM et al. Histone methyltransferase gene SETD2 is a novel tumor suppressor gene in clear cell renal cell carcinoma. Cancer Res 2010; 70: 4287-4291.

7 Pfister SX, Ahrabi S, Zalmas LP, Sarkar S, Aymard F, Bachrati CZ et al. SETD2dependent histone $\mathrm{H} 3 \mathrm{~K} 36$ trimethylation is required for homologous recombination repair and genome stability. Cell Rep 2014; 7: 2006-2018.

8 Carvalho S, Vítor AC, Sridhara SC, Martins FB, Raposo AC, Desterro JM et al. SETD2 is required for DNA double-strand break repair and activation of the p53mediated checkpoint. Elife 2014, e02482.

9 Kanu N, Grönroos E, Martinez P, Burrell RA, YiGoh X, Bartkova J et al. SETD2 loss-offunction promotes renal cancer branched evolution through replication stress and impaired DNA repair. Oncogene 2015; 34: 5699-5708.

10 Guièze R, Wu CJ. Genomic and epigenomic heterogeneity in chronic lymphocytic leukemia. Blood 2015; 126: 445-453.

11 Catovsky D, Richards S, Matutes E, Oscier D, Dyer MJ, Bezares RF et al. Assessment of fludarabine plus cyclophosphamide for patients with chronic lymphocytic leukaemia (the LRF CLL4 Trial): a randomised controlled trial. Lancet 2007; 370: 230-239.

12 Hallek M, Fischer K, Fingerle-Rowson G, Fink AM, Busch R, Mayer J et al. Addition of rituximab to fludarabine and cyclophosphamide in patients with chronic lymphocytic leukaemia: a randomised, open-label, phase 3 trial. Lancet 2010; 376: 1164-1174.

13 Oscier D, Wade R, Davis Z, Morilla A, Best G, Richards S et al. Prognostic factors identified three risk groups in the LRF CLL4 trial, independent of treatment allocation. Haematologica 2010; 95: 1705-1712.

14 Parry M, Rose-Zerilli MJ, Gibson J, Ennis S, Walewska R, Forster J et al. Whole exome sequencing identifies novel recurrently mutated genes in patients with splenic marginal zone lymphoma. PLoS One 2013; 8: e83244.

15 Parker H, Rose-Zerilli M, Parker A, Chaplin T, Chen X, Wade R et al. 13q deletion anatomy and disease progression in patients with chronic lymphocytic leukemia. Leukemia 2011; 25: 489-497.

16 Rose-Zerilli M, Forster J, Parker H, Parker A, Rodriguez A, Chaplin T et al. ATM mutation rather than $\mathrm{BIRC} 3$ deletion and/or mutation predicts reduced survival in 11q-deleted chronic lymphocytic leukemia, data from the UK LRF CLL4 trial. Haematologica 2014; 99: 736-742.

17 Edelmann J, Saub J, Ibach S, Holzmann K, Tausch E, Bloehdorn J et al. High resolution genomic profiling of primary "ultra high risk" and refractory chronic lymphocytic leukemia: results from the CLL2O Trial. Blood (ASH Annual Meeting Abstracts) 2014; 120: 3288.

18 Edelmann J, Holzmann K, Miller F, Winkler D, Bühler A, Zenz T et al. Highresolution genomic profiling of chronic lymphocytic leukemia reveals new recurrent genomic alterations. Blood 2012; 120: 4783-4794.

19 Knight SJ, Yau C, Clifford R, Timbs AT, Sadighi Akha E, Dréau HM et al. Quantification of subclonal distributions of recurrent genomic aberrations in paired pretreatment and relapse samples from patients with B-cell chronic lymphocytic leukemia. Leukemia 2012; 26: 1564-1575.

20 Clifford R, Louis T, Robbe P, Ackroyd S, Burns A, Timbs AT et al. SAMHD1 is mutated recurrently in chronic lymphocytic leukemia and is involved in response to DNA damage. Blood 2014; 123: 1021-1031.

21 Parry M, Rose-Zerilli MJJ, Ljungström V, Gibson J, Wang J, Walewska R et al. Genetics and prognostication in splenic marginal zone lymphoma: revelations from deep sequencing. Clin Cancer Res 2015; 21: 4174-4183.

22 Robbe P, Clifford R, Timbs A, Burns A, Titsias M, Cabes M et al. Comprehensive Genome-wide Analysis of CLL Samples from UK 1st Line and Relapsed/Refractory Clinical Trials. EHA Annual Meeting Abstracts, 2013:EHA18ABSSUB-4559.

23 Landau DA, Tausch E, Taylor-Weiner AN, Stewart C, Reiter JG, Bahlo J et al. Mutations driving CLL and their evolution in progression and relapse. Nature 2015; 526: 525-530.

24 An Q, Wright SL, Konn ZJ, Matheson E, Minto L, Moorman AV et al. Variable breakpoints target PAX5 in patients with dicentric chromosomes: a model for the basis of unbalanced translocations in cancer. Proc Natl Acad Sci USA 2008; 105: 17050-17054.
25 Ouillette P, Fossum S, Parkin B, Ding L, Bockenstedt P, Al-Zoubi A et al. Aggressive chronic lymphocytic leukemia with elevated genomic complexity is associated with multiple gene defects in the response to DNA double-strand breaks. Clin Cancer Res 2010; 16: 835-847.

26 Mermel CH, Schumacher SE, Hill B, Meyerson ML, Beroukhim R, Getz G. GISTIC2.0 facilitates sensitive and confident localization of the targets of focal somatic copy-number alteration in human cancers. Genome Biol 2011; 12: R41.

27 Forbes SA, Bindal N, Bamford S, Cole C, Kok CY, Beare D et al. COSMIC: mining complete cancer genomes in the Catalogue of Somatic Mutations in Cancer. Nucleic Acids Res 2011; 39: D945-D950.

28 Puente XS, Beà S, Valdés-Mas R, Villamor N, Gutiérrez-Abril J, Martín-Subero J et al. Non-coding recurrent mutations in chronic lymphocytic leukaemia. Nature 2015; 526: 519-524.

29 Carter SL, Cibulskis K, Helman E, McKenna A, Shen H, Zack T et al. Absolute quantification of somatic DNA alterations in human cancer. Nat Biotech 2012; 30: 413-421.

30 Landau DA, Carter SL, Stojanov P, McKenna A, Stevenson K, Lawrence MS et al. Evolution and impact of subclonal mutations in chronic lymphocytic leukemia. Cell 2013; 152: 714-726.

31 Edmunds JW, Mahadevan LC, Clayton AL. Dynamic histone H3 methylation during gene induction: $\mathrm{HYPB} / \mathrm{Setd} 2$ mediates all $\mathrm{H} 3 \mathrm{~K} 36$ trimethylation. $E M B O$ J 2008; 27: 406-420.

32 Yoh SM, Lucas JS, Jones KA. The Iws1:Spt6:CTD complex controls cotranscriptional mRNA biosynthesis and HYP/Setd2-mediated histone H3K36 methylation. Gene Dev 2008; 22: 3422-3434.

33 Wagner EJ, Carpenter PB. Understanding the language of Lys36 methylation at histone H3. Nat Rev Mol Cell Biol 2012; 13: 115-126.

34 Li F, Mao G, Tong D, Huang J, Gu L, Yang W et al. The histone mark H3K36me3 regulates human DNA mismatch repair through its interaction with MutSa. Cell 2013; 153: 590-600.

35 Kolasinska-Zwierz P, Down T, Latorre I, Liu T, Liu XS, Ahringer J. Differential chromatin marking of introns and expressed exons by H3K36me3. Nat Genet 2009; 41: 376-381.

36 Sun $\mathrm{XJ}$, Wei J, Wu XY, Hu M, Wang L, Wang $\mathrm{HH}$ et al. Identification and characterization of a novel human histone $\mathrm{H} 3$ lysine 36-specific methyltransferase. J Biol Chem 2005; 280: 35261-35271.

37 Huether R, Dong L, Chen X, Wu G, Parker M, Wei L et al. The landscape of somatic mutations in epigenetic regulators across 1,000 paediatric cancer genomes. Nat Commun 2014; 3: 3630.

38 Mar BG, Bullinger LB, McLean KM, Grauman PV, Harris MH, Stevenson K et al. Mutations in epigenetic regulators including SETD2 are gained during relapse in paediatric acute lymphoblastic leukaemia. Nat Commun 2014; 5: 3469.

39 Lee WP, Stromberg MP, Ward A, Stewart C, Garrison EP, Marth GT. MOSAIK: a hash-based algorithm for accurate next-generation sequencing short-read mapping. PLoS One 2014; 9: e90581.

40 Lawrence MS, Stojanov P, Polak P, Kryukov GV, Cibulskis K, Sivachenko A et al. Mutational heterogeneity in cancer and the search for new cancerassociated genes. Nature 2013; 499: 214-218.

41 Kulis M, Heath S, Bibikova M, Queirós AC, Navarro A, Clot G et al. Epigenomic analysis detects widespread gene-body DNA hypomethylation in chronic lymphocytic leukemia. Nat Genet 2012; 44: 1236-1242.

42 Rhodes DR, Kalyana-Sundaram S, Mahavisno V, Varambally R, Yu J, Briggs BB et al. Oncomine 3.0: genes, pathways, and networks in a collection of 18,000 cancer gene expression profiles. Neoplasia 2007; 9: 166-180.

43 Xie P, Tian C, An L, Nie J, Lu K, Xing G et al. Histone methyltransferase protein SETD2 interacts with $\mathrm{p} 53$ and selectively regulates its downstream genes. Cell Signal 2008; 20: 1671-1678.

44 Gross AM, Orosco RK, Shen JP, Egloff AM, Carter H, Hofree M et al. Multi-tiered genomic analysis of head and neck cancer ties TP53 mutation to 3p loss. Nat Genet 2014; 46: 939-943.

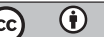

This work is licensed under a Creative Commons Attribution 4.0 International License. The images or other third party material in this article are included in the article's Creative Commons license, unless indicated otherwise in the credit line; if the material is not included under the Creative Commons license, users will need to obtain permission from the license holder to reproduce the material. To view a copy of this license, visit http://creativecommons.org/licenses/ by/4.0/

(c) The Author(s) 2016

Supplementary Information accompanies this paper on the Leukemia website (http://www.nature.com/leu) 